\title{
The Establishment of Peripheral Sensory Arbors in the Leech: In Vivo Time-Lapse Studies Reveal a Highly Dynamic Process
}

\author{
Huajun Wang and Eduardo R. Macagno \\ Department of Biological Sciences, Columbia University, New York, New York 10027
}

Pressure-sensitive $(P)$ neurons located in the leech CNS form elaborate terminal arbors in the body wall of the animal during mid-embryogenesis. In the experiments discussed here, arbor development in the target region was studied in intact, unanesthetized leech embryos using time-lapse video microscopy of individual, fluorescently stained $P$ neurons. Analysis of timelapse recordings made over a period of several days revealed that arbor formation is a very dynamic process. At any particular time, most high-order terminal branches were either extending or retracting, in approximately equal numbers and at very similar rates. Many branches underwent several rounds of extension and retraction every hour. Net arbor growth occurred at a much lower rate than the extension and retraction rates of individual branches. Process retraction sometimes resulted in an apparent change in the topological order of processes. Significantly, the initiation of new branches was restricted to a few locations along the parent process, which were termed "hot spots." Moreover, the capacity to generate high-order branches correlated with parent process stability.

The target region of the growing $\mathrm{P}$ cell arbor in the body wall was subsequently examined using confocal microscopy in fixed preparations. The arbor expanded between the longitudinal and circular muscle layers, a region occupied by small unidentified cells. Simultaneous imaging of the dye-labeled terminal arbor and the surrounding tissue at two different wavelengths suggested that the high-order processes were navigating around these cells, which sometimes forced the growing processes to assume a bent form.

These observations suggest that the formation of the $P$ cell arbor can be best described as a "dynamically unstable" process that is constrained by interactions with its environment.

Key words: axon outgrowth; topological order; dynamic instability; time-lapse imaging; video microscopy; Hirudo medicinalis
Since the pioneering neuroanatomical work of Santiago Ramon y Cajal, neurobiologists have sought to identify the mechanisms that generate the extraordinary shapes of neurons. Neuronal shapes are so consistent that neurons can be reliably classified according to conserved features of their arborization patterns. This has been the basis for extensive literature describing the varied and elaborate morphologies that define different types of neurons (Peters and Jones, 1984). Despite much thought and experimentation, the underlying mechanisms that generate such individual shapes are mostly unknown.

Neuronal morphogenesis is undoubtedly very complicated and can be a protracted process. A typical cortical neuron, for example, can take several weeks or months to achieve its complex mature shape (Miller, 1981, 1986). During development, neurons encounter a variety of cellular and acellular substrates as their projections extend within and beyond the nervous system. Neurons attain their individual morphologies by playing out an innate growth program in an instructive environment that modulates and refines the expression of this program.

Interestingly, this interplay of intrinsic program and extrinsic interactions does not appear to yield unique results. When identified neurons were examined in isogenic organisms, for example, significant variations in branching patterns were observed between specimens (Macagno et al., 1973; Levinthal et al., 1976). A

\footnotetext{
Received Sept. 6, 1996; revised Jan. 16, 1997; accepted Jan. 23, 1997.

We thank Dr. Laura Wolszon for critical reading and suggestions for improvement of this manuscript.

Correspondence should be addressed to Eduardo R. Macagno, 1011 Fairchild Center, Columbia University, New York, NY 10027.

Copyright (C) 1997 Society for Neuroscience $0270-6474 / 97 / 172408-12 \$ 05.00 / 0$
}

possible conclusion from observations such as these is that neuronal growth must have both highly regulated and stochastic components, the first yielding the conserved features we recognize as defining the identity of the cell, and the latter the more variable, finer details.

It has been proposed that conserved features may reflect a neuron's intrinsic program (Solomon, 1979; Montague and Friedlander, 1989), but in some instances this would be incorrect. For example, the primary branching pattern of the AP neuron in the dorsal body wall of the leech is highly stereotyped, but this pattern has been shown to result from the AP cell using another cell as a template, without which it is incapable of generating its normal pattern (Gan and Macagno, 1995b). Because such cell-cell interactions clearly play a critical role in a neuron's expression of specific anatomical features, examining how a neuron grows in its normal environment can provide important insight into its functions.

In the studies reported here, we used in vivo time-lapse fluorescence microscopy of an identified neuron to make a detailed analysis of the spatial and temporal dynamics of terminal arbor formation in the intact animal. In the past decade, a number of time-lapse studies of individual neurites in their normal context (mainly in dissected preparations or in brain slices) have demonstrated the dynamic nature of neuronal process outgrowth in several systems (Harris et al., 1987; O’Connor et al., 1990; Kaethner and Stuermer, 1992; Myers and Bastiani, 1993; Halloran and Kalil, 1994; O'Rourke et al., 1994). Most of these studies, unlike those reported here, examined either axons projecting toward the CNS or projections within the CNS. Temporal changes in arbor 


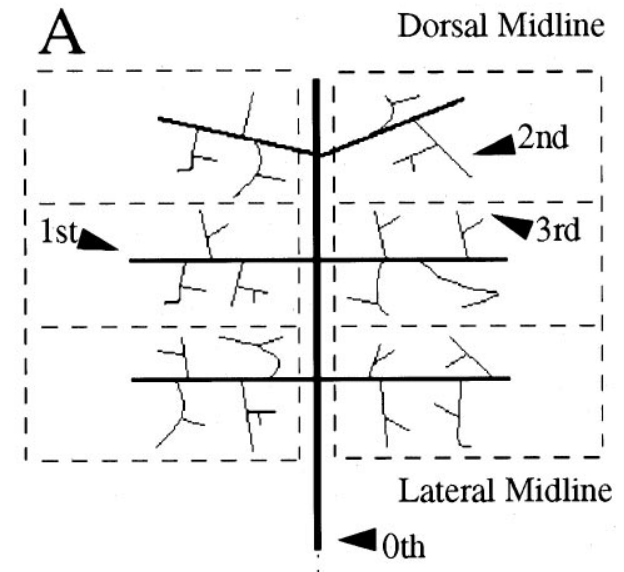

\section{Cell Body}
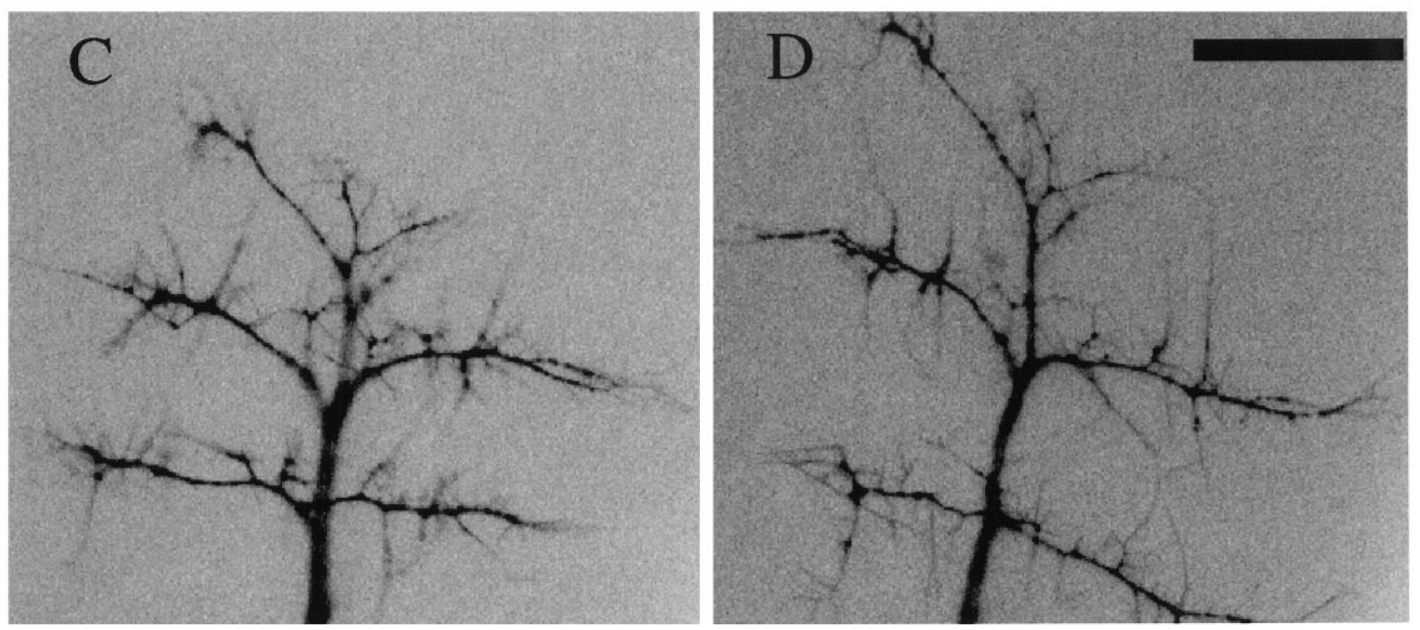

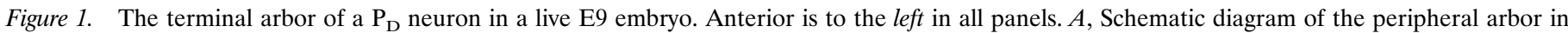

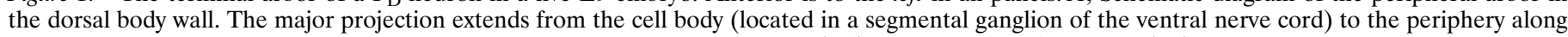

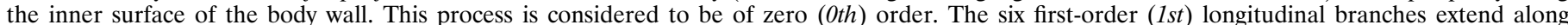

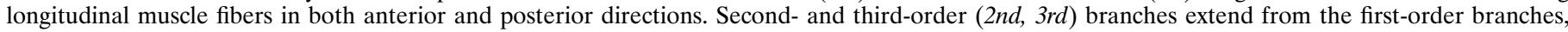

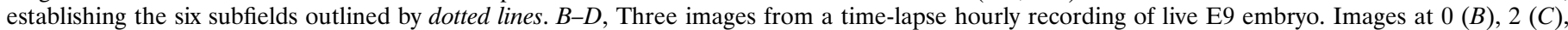

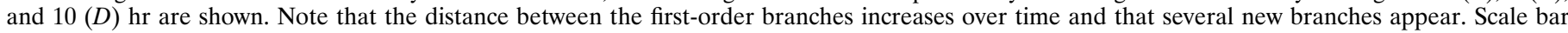
for $B-D, 100 \mu \mathrm{m}$.

formation in the periphery have been explored mainly in the formation of the neuromuscular junction, an area in which Lichtman and colleagues have developed techniques for visualizing the same junction at different times in the intact animal (Lichtman et al., 1987). Interestingly, such studies have shown that a neuromuscular junction can expand to a bigger area as the muscle grows, without addition of branches (Balice-Gordon and Lichtman, 1990).

The neuron under study here was the pressure-sensitive $(\mathrm{P})$ cell, whose cell body lies within the CNS but which arborizes in the body wall. These neurons have been the subject of extensive anatomical and electrophysiological studies, and their electrical properties and receptive fields are well characterized (Nicholls and Baylor, 1968; Muller and McMahan, 1976; Yau, 1976; Sargent et al., 1977).

There are two bilaterally symmetric pairs of $\mathrm{P}$ cells in most leech segmental ganglia, each pair innervating either ventral $\left(\mathrm{P}_{\mathrm{V}}\right)$ or dorsal $\left(\mathrm{P}_{\mathrm{D}}\right)$ skin. They project to the ipsilateral body wall through the nerve roots of three adjacent segmental ganglia, forming a major receptive field in the central segment and minor fields in the adjacent segments. The major field consists of three anterior and three posterior first-order branches that arborize profusely, establishing six subfields with well defined boundaries (see Fig. 1).

From embryonic day (E) 9 (embryogenesis lasts $30 \mathrm{~d}$ at $23^{\circ} \mathrm{C}$ ) to early E11, when most of these time-lapse studies in intact embryos were carried out, we found the terminal field to be a very complicated and dynamic structure. With several hundred processes changing at any particular time and the boundaries between subfields being formed, we were able to document the initiation, extension, and retraction of many high-order processes. In addition, using confocal microscopy in fixed preparations, we have examined the relationship between the growing arbor and cellular components of the target region in the body wall. 


\section{MATERIALS AND METHODS}

Animals. Hirudo medicinalis embryos were obtained from our laboratory colony and maintained at $23^{\circ} \mathrm{C}$ in artificial spring water (dilute Instant Ocean, $0.5 \mathrm{gm} / \mathrm{l})$.

Labeling neurons with DiI. Embryos were anesthetized with $9 \%$ ethanol in Wenning's solution containing (in $\mathrm{mM}$ ): 40 DL-malic acid, $4 \mathrm{KCl}, 10$ succinic acid, 10 Tris- $\mathrm{HCl}$, and $1.8 \mathrm{CaCl}_{2}, \mathrm{pH} 7.4$ (Wenning, 1987). A small cut in the skin over the experimental ganglion was made to visualize the P cells. Glass microelectrodes with fine tips were pulled on a Sutter P-87 puller, and their tips were bent in a microforge to an angle $\sim 45^{\circ}$ relative to the electrode shaft. They were filled with a $1 \%$ solution of the lipophilic fluorescent dye 1,1'-dioctadecyl-3,3,3',3'-tetramethylindocarbocyanine perchlorate (DiI; Molecular Probes, Eugene, OR) in $100 \%$ methylene chloride. The tip of the electrode was then placed on or just within the plasma membrane of identified cells under a $40 \times$ water immersion objective. One or two pulses of depolarizing current $(1 \mathrm{nA})$ were applied to eject a small amount of dye. The embryos were then returned to artificial spring water and kept in darkness for $\sim 12 \mathrm{hr}$ to allow the dye to diffuse to the fine processes in the terminal field.

Imaging the terminal fields. For imaging, intact embryos were placed in artificial spring water without anesthetic in a $30 \mathrm{~mm}$ plastic dish, the bottom of which was replaced with a thin glass coverslip. To reduce movement, the embryos were held on the coverslip mechanically, using a sterile, thin nylon mesh that was placed over the embryo and held down with small weights. The terminal fields of $P$ cells were imaged through the coverslip and through the skin using a cooled charge-coupled device camera mounted on a Nikon inverted microscope equipped with highresolution $10 \times$ and $40 \times$ oil immersion objectives. To reduce photodamage and dye bleaching, the preparation was illuminated for only $100 \mathrm{msec}$ while taking each image.

The images of the terminal fields were stored and displayed using software developed originally for calcium imaging (Regehr and Tank, 1992) and modified for our use. These images were then transferred to a Unix workstation and analyzed using the latest version (III) of the CARTOS software package developed at Columbia University (Allen and Levinthal, 1990). Low- and high-threshold levels were selected empirically to reproduce the image visualized through the eyepieces. All of the pixel values were digitally inverted so the terminal fields could be displayed as gray-scale images on a nearly white background (see Fig. 1). No further image processing was done for most of the analyses.

Selection of appropriate time intervals. At first, we imaged terminal fields at approximately hourly intervals. However, because of the complexity of the terminal fields, the hourly recordings did not allow us to unambiguously identify every, or even most, processes over time. Therefore, the fields were imaged every minute, because test recordings done every $6 \mathrm{sec}$ suggested that $1 \mathrm{~min}$ intervals provided sufficient resolution to follow the dynamics of most processes. This necessitated holding the animal more tightly on the coverslip. As a result, movement of the skin was minimized, and the whole field was flatter so the entire branching pattern could be imaged in one focal plane at low power.

Because embryos were not rigidly affixed to the coverslip, they had to be repositioned, and the optics were refocused frequently during imaging. However, this arrangement ensured that the images were taken under the most natural conditions for the animal. Most time-lapse recordings were terminated by E12 because of excessive movement.

Analysis of the time-lapse series. Single images from a recorded series do not allow the resolution of all details of arbor growth. However, the temporal integration obtained when time-lapse series are played back at high speed can be quite helpful in resolving details (Myers and Bastiani, 1993). CARTOS-III allows the replaying of any segment of the time-lapse series at video rate $(\sim 30$ frames per second), which helps greatly in resolving the details of the images. As a result, we were capable of resolving the dynamics of most processes in the recorded area.

To obtain quantitative data, the apparent length (L) of a process of interest was traced from its initiation point to its tip in every frame. The function $L(t)$ then gave us information about the extension and retraction of the process. Any successive local minimum (L1), local maximum (L2), and then local minimum $(L 3)$ of $L(t)$ at times $t_{1}, t_{2}$, and $t_{3}$ corresponds to one round of extension and retraction; $(L 2-L 1) /\left(t_{2}-t_{1}\right)$ and $(L 3-$ $L 2) /\left(t_{3}-t_{2}\right)$ give the average extension and retraction rate. Because of embryo movement and the limited resolution of the digitized images, we could not resolve accurately differences below 1 or 2 pixels, which translated to approximately 1 or $2 \mu \mathrm{m}$ when imaging at low magnification. Therefore, we only considered cases in which processes grew $>2 \mu \mathrm{m}$ as being an "extension." The "instantaneous" rate can also be calculated from differences in $L$ in successive frames. The maximum of this rate is defined as the peak rate.

Because the terminal field is a very complicated structure with several hundred processes, it was not feasible to trace all of them over time. In general, we focused on high-order processes and randomly selected some number of them to trace, without regard for their order, length, or history. Because a process could have a bent structure and its topological order could change (see Results), it was not always clear for the branched processes (which had multiple free ends) which end belonged to the parent branch and which were higher-order processes. We usually assumed the longest and least bent process to be the parent branch. When a bias was occasionally introduced in selecting processes for analysis, this is indicated in the corresponding Results.

In cases in which the animal did not move significantly, we were able to compare two (not necessarily successive) frames by the following method. The first frame (time $t_{1}$ ) was changed to an intensity-scaled color image by converting each pixel value, $p_{1}$, to the color red-green-blue value $\left(p_{1}\right.$, $0,0)$. The second frame (time $t_{2}>t_{1}$ ) was then changed by converting $p_{2}$ to $\left(0, p_{2}, p_{2}\right)$. The two images were then digitally combined. In the resulting composite image, a pixel was the original gray value if it did not change from $t_{1}$ to $t_{2}$. However, if the pixel value was significantly different between the two frames, it appeared as red (if $p_{1} \gg p_{2}$ ) or cyan (if $p_{2} \gg$ $\left.p_{1}\right)$. This method allowed us to visualize in the combined image all of the changes between two frames. In particular, processes that extended between $t_{1}$ and $t_{2}$ appeared in red $\left(p_{1} \gg p_{2}\right.$, because we were using the inverted intensities of the fluorescent images), and those that retracted in this interval appeared in cyan.

Controls. To determine whether dye filling and time-lapse imaging affected $\mathrm{P}$ cell development, we compared cells imaged over several days with cells stained at daily intervals and fixed immediately without imaging. Ten cells were stained at early E9 and then imaged at late E9, E10, and E11. Their arbors (not shown) were comparable in extent and complexity to those of fixed preparations (Gan and Macagno, 1995a), indicating that $\mathrm{P}$ cells continued to develop normally after staining and imaging as described above.

Confocal imaging of the target region. For imaging dye-filled cells together with their peripheral environments, preparations were fixed in $4 \%$ paraformaldehyde after DiI injection and kept for 10-14 d at room temperature to allow the dye to diffuse throughout the arbor of the cell. In some preparations, Lucifer yellow was added to the fixative (final concentration, $0.01 \%$ ) to enhance background fluorescence at shorter wavelengths. Subsequently, preparations were washed, cleared, and mounted in $100 \%$ glycerol. Using a laser confocal microscope (Bio-Rad, MRC-600) with a $63 \times$ oil immersion objective, serial images were collected by optically sectioning at $1 \mu \mathrm{m}$ intervals. To image $\mathrm{P}$ cells and their environments, preparations were excited at $488 \mathrm{~nm}$, and a dual channel filter set was used to collect emitted light at $520 \mathrm{~nm}$ for background (or Lucifer yellow) fluorescence and at $585 \mathrm{~nm}$ for DiI. The addition of Lucifer yellow resulted in more contrast in the shorter wavelength channel. Because the selectivity of Lucifer yellow staining in these conditions is not known, we compared preparations with and without this dye when attempting to identify cellular elements in the target region of the body wall.

It should be noted that in all of these time-lapse recordings, the microscope objective was focused so that most of the higher-order processes were in focus, whereas the main projection and first-order branches were slightly out of focus (thus appearing thicker than they really are). High magnification confocal images such as those in Figure 2 indicate that the main projection and the first-order branches are approximately 2-3 $\mu \mathrm{m}$ in diameter, whereas the thickness of most of the high-order processes is below the resolution of the light microscope.

\section{RESULTS}

The main projection of a $P_{D}$ neuron grows laterally from the ganglion through the posterior root, first through ventral territory where it does not form any permanent branches, and then through dorsal territory where it extends six longitudinal branches at specific locations (Fig. 1A) (Gan and Macagno, 1995a). By early $\mathrm{E} 9$, the earliest stage examined in the work reported here, the six first-order branches, as well as some of the second-order processes, were already generated (Fig. $1 B$ ). Hence, the observations reported here, carried out between early E9 and late E11 (see Figs. $1 B-D, 8)$, describe mainly the initiation, retraction, and 

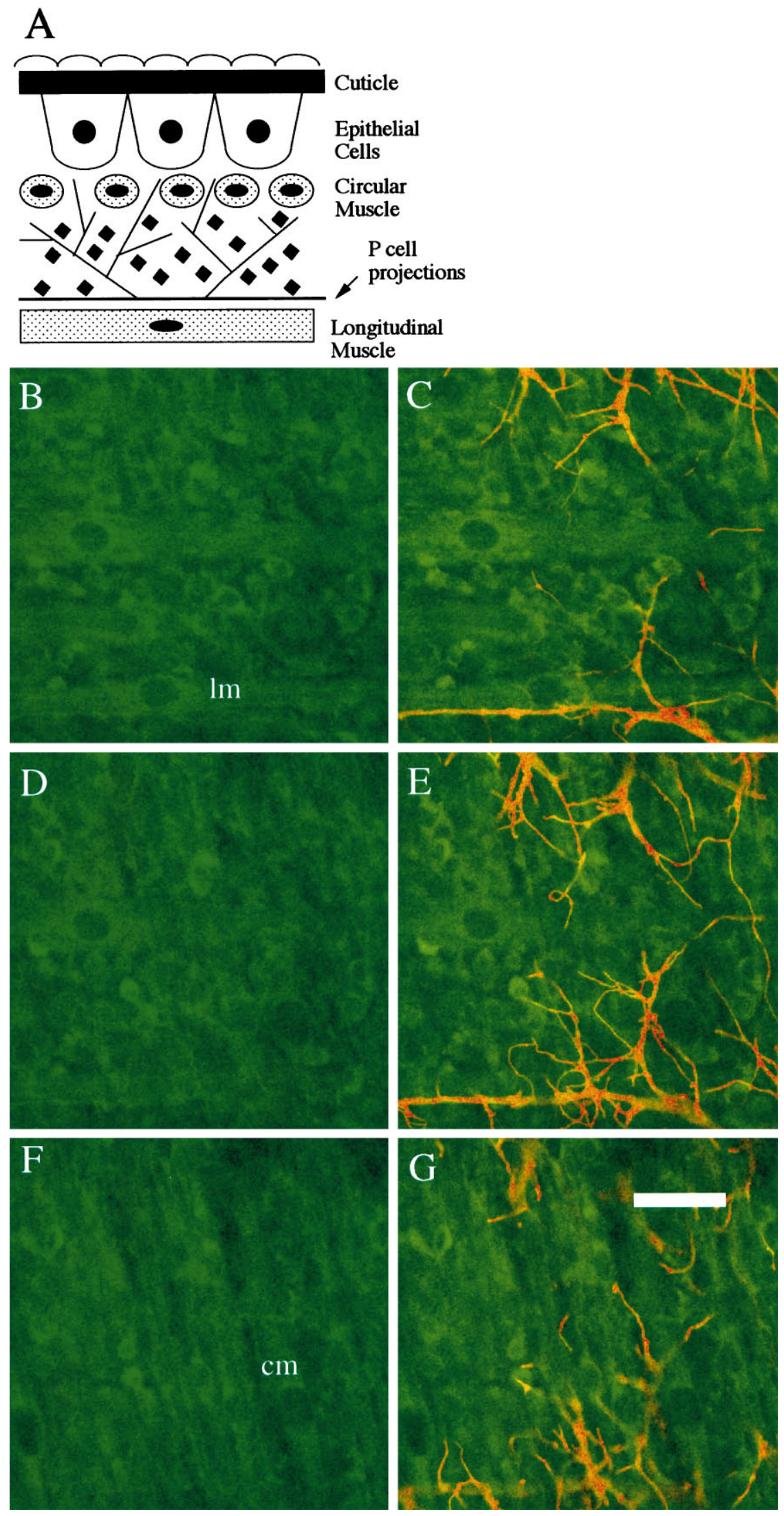

Figure 2. Confocal images of the body wall and P cell terminals in a fixed preparation. Anterior is to the left. $A$, Schematic diagram of a cross section of the dorsal body wall along the antero-posterior axis of the leech. The layers represented include the cuticle, the large epithelial cells, the circular muscle layer, the longitudinal muscle layer, and a layer of small undefined cells (diamonds) that is interposed between the two muscle layers. The arbor of the $\mathrm{P}$ cell is shown extending between the two muscle layers. $B-G$, Confocal images of part of the terminal field in one preparation. $B, D$, and $F$ show green channel images (see Materials and Methods) taken at 20,15, and $10 \mu \mathrm{m}$ below the body wall surface. Some longitudinal $(\mathrm{lm})$ and circular $(\mathrm{cm})$ muscle fibers are indicated in $B$ and $F$, respectively. $C$, $E$, and $G$ show the same focal planes but with the green and red channels added to display both the target tissue and the dye-filled P cell. Scale bar for $B-G, 20 \mu \mathrm{m}$. stabilization of second-, third-, and fourth-order branches in the terminal arbor of the $\mathrm{P}$ cell.

We first documented that as the terminal field of the $\mathrm{P}$ cell increased in extent and complexity, it also underwent an expansion in response to the embryo increasing in diameter and length. Figure $1 B-D$ shows a series of three images several hours apart, 
beginning at early E9. Note the increasing distances between the first-order longitudinal branches with time. This expansion continued throughout E10 (not shown) and even into adulthood, but it was much slower after E9. To determine whether this expansion correlated with the overall growth of the embryo, we measured in unstained control embryos the distance from the center of a ganglion to the lateral edge of the germinal plate. During the first $12 \mathrm{hr}$ of E9, it increased by $\sim 40 \%$, but afterward it generally increased more slowly, only by $\sim 10 \%$ every $12 \mathrm{hr}$ until E12. These numbers are consistent with the lateral arbor expansion observed in time-lapse recordings being defined by overall embryonic growth.

\section{The $P_{D}$ cell arbor expands between the longitudinal and circular muscle layers}

The body wall of the adult leech is comprised of several layers of different tissues, including four distinctive sets of muscle fibers. The most internally located muscles are the longitudinal fibers, oriented anteroposteriorly, followed by two layers of oppositely oriented oblique muscles, a layer of circular muscles, and an outermost layer of annulus erector muscles in each annulus. At the embryonic stages we studied in these experiments, only the longitudinal and circular muscles were clearly differentiated and visible in the body wall; the region to be occupied later by the oblique muscles contained only small cells of undetermined type (shown schematically in Fig. $2 A$ ).

Examination by focusing through both live and fixed preparations with the light microscope suggested that the expanding arbor of the $\mathrm{P}_{\mathrm{D}}$ neuron might be confined to a narrow layer within the body wall, at a slightly more external plane than either the major projection or the first-order branches. To confirm this, we obtained serial optical sections of fixed preparations with DiIinjected $\mathrm{P}_{\mathrm{D}}$ neurons, using endogenous shorter wavelength (green channel) fluorescence to visualize the components of the body wall and longer wavelength (red channel) fluorescence to visualize the dye-filled cell in the same preparation. In some cases, Lucifer yellow was added to the fixative to enhance the background fluorescence (see Materials and Methods). The results from observations of 20 preparations (E9-E11 embryos) are summarized in the schematic shown in Figure $2 A$; examples from one optical series are shown in Figure $2 B-G$.

Although unstained preparations had a poor signal-to-noise ratio, we were able to recognize readily some of the longitudinal and circular muscle fibers by their distinct shapes. In the fixed preparations, the longitudinal muscle layer was found to be $\sim 20$ $\mu \mathrm{m}$ from the body wall surface, and the circular muscle layer only $\sim 10 \mu \mathrm{m}$, leaving a space between them approximately 5-10 $\mu \mathrm{m}$ thick (Fig. 2B,D,F). The main projection of the $\mathrm{P}_{\mathrm{D}}$ cell was observed to extend along the inner surface of the body wall to dorsal territory, where the six first-order branches (see Fig. 1) were formed and appeared to grow among and parallel to longitudinal muscle fibers (see Fig. 2C). A similar observation was made in a previous study using the Laz10-1 monoclonal leech muscle antibody (Gan, 1995). Higher-order processes then extended into and branched profusely in the region between the two muscle layers (Fig. 2E), with some branches reaching and growing along and between the circular fibers (Fig. $2 E$ ) toward the body wall surface (see schematic, Fig. $2 A$ ).

Interposed between the two muscle layers were many small cells of undetermined type, approximately 3-5 $\mu \mathrm{m}$ in diameter. These cells were distributed somewhat irregularly both horizontally and vertically. It is known that another muscle layer, the oblique muscle layer, starts to develop between the circular and longitudinal muscle layers at E12 (Jellies and Kristan, 1988, 1991). Therefore, it is likely that at least some of these interposed cells are myoblasts. Because of the low contrast of the images in the green channel, it is hard to get an accurate measurement of the number of such cells, but a rough estimate yields at least one cell per $250-500 \mu \mathrm{m}^{3}$.

Dual-channel images indicate that $\mathrm{P}$ cell processes grew between these interposed cells in a manner that suggests a stochastic process (see Fig. $2 E, G$ ). In fact, that branches sometimes appeared to be bent rather than straight may be a reflection of nothing more than having to grow around these small cells. Whether these cells interact in any specific manner with the growing $\mathrm{P}$ cell cannot be discerned from our observations.

\section{Extension and retraction of processes is a pervasive feature of $P$ cell arbor growth}

A striking conclusion from our observations of the developing terminal field of $\mathrm{P}_{\mathrm{D}}$ neurons was that most second- and higherorder branches were in a constantly dynamic state from E9 to E11, even those that were eventually stabilized. This can be seen readily in Figure $3 C$, which shows a colorized composite (see Materials and Methods) of two images taken 20 min apart; the images are shown individually in Figure 3, $A$ and $B$. The sections of processes that had extended or retracted during this interval are displayed in red and cyan, respectively. Similar degrees of change were observed in the analyses of $\sim 200 \mathrm{hr}$ of recordings of the terminal fields of 42 different $P_{D}$ neurons, leading to the conclusion that many changes occurred within a time scale of minutes. Figure $3 D$ shows a composite from another recording that combined two frames 3 min apart. It is clear that, even within such a short time interval, the terminal field was very dynamic.

In general, few processes appeared to be stationary for longer than $\sim 30 \mathrm{~min}$. To quantify this observation, we analyzed 100 randomly selected second- and third-order processes, 25 from each of four different neurons, for a period of $30 \mathrm{~min}$ within a longer time-lapse sequence. These included approximately 5-10\% of the total population of the processes of each neuron. Of the 100 processes analyzed, 68 were found to extend or retract within 10 min, 18 within $10-20 \mathrm{~min}$, and 11 within $30 \mathrm{~min}$. Only 3 processes did not clearly change within $30 \mathrm{~min}$, although they had when examined $1 \mathrm{hr}$ later (Fig. 4).

\section{Branch retraction at bifurcation points can give rise to bent processes and revised rank order}

When examining the terminal segments of higher-order processes, we found that most extended in straight lines, but some displayed distinct bends. To determine how these were generated, we examined 20 bent processes from four separate recordings. We found that 10 extended along nonlinear paths as they were generated. These bent processes may have resulted from growth around obstacles or between cells (see Fig. 2G). The other 10 bent processes, however, were the result of the retraction of one of the two processes at a bifurcation. Figure 5 shows an example of a bifurcation becoming a bent process through retraction.

An interesting consequence of process retraction is that the apparent rank order of a branch can change. In the case illustrated in Figure 5, for example, a third-order branch (number 2) becomes second-order as the original second-order process (number 1) retracts to the branch point. 

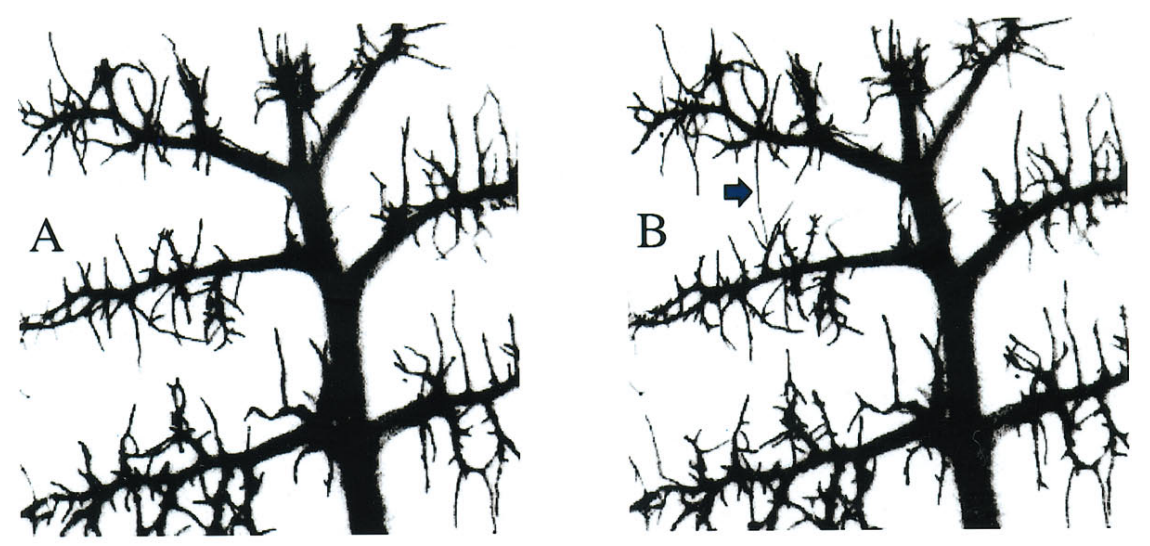

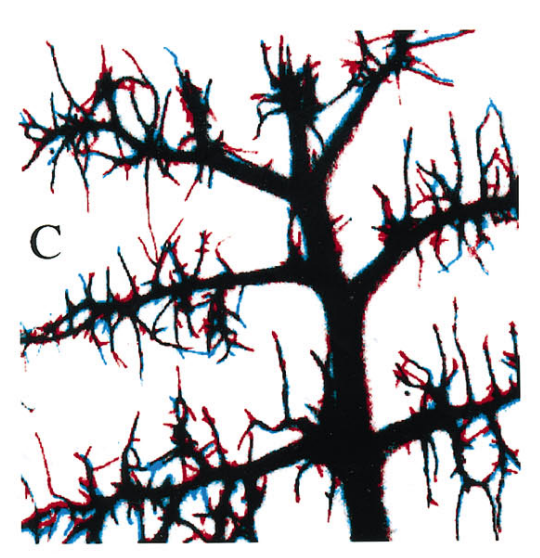

Figure 3. Changes in the $\mathrm{P}$ cell arbor that occurred within a span of $20 \mathrm{~min}$. $A$, $B$, Two images, $20 \mathrm{~min}$ apart, from a time-lapse series of a live $\mathrm{E} 9$ embryo. $C$, Color composite of the two images in $A$ and $B$ (see Materials and Methods), illustrating the large changes in the arbor that occurred in this short time interval. The segments of the processes that are newly extended during this period appear in red, whereas those segments that were present in $A$ but had been retracted in $B$ are shown in cyan. $D$, Higher-magnification color composite of two images taken $3 \mathrm{~min}$ apart, from another time-lapse series. It is clear that even in such a short period the terminal field can display significant changes. Anterior is to the left. Scale bars: $A-C, 100$ $\mu \mathrm{m} ; D, 50 \mu \mathrm{m}$.

\section{Branches reinitiate growth primarily at previous initiation sites}

When playing back time-lapse recordings at high speed, we had the distinct impression that branches were generated at a relatively limited number of locations along the parent process, at positions where branches had been initiated and retracted previously. To obtain a more quantitative assessment of this phenomenon, we analyzed third-order branch initiation by a second-order process during an $8 \mathrm{hr}$ period. At the beginning of the observation period, this process had no branches, and none formed during the first $3 \mathrm{hr}$. However, 33 branches were initiated over the next $5 \mathrm{hr}$ (Fig. 6). Most of these were short and were retracted within a few minutes after they were initiated (Fig. 7A), their average lifetime being just $8.1 \pm 6.2 \mathrm{~min}$. (mean $\pm \mathrm{SD}$ ), and their average peak length $9.2 \pm 5.2 \mu \mathrm{m}$. However, the plot of such branch initiations versus time, presented in Figure $6 B$, clearly shows that most of the 33 processes emanated from three locations along the parent process, indicating the presence of "hot spots" where processes were most likely to appear.

To test this "hot spots" hypothesis in a different way, we arbitrarily selected, from images of two different neurons, 28 branch initiation events that took place toward the end of the recording period. We then looked at previous images in the series (up to $150 \mathrm{~min}$ earlier) for the presence of earlier branch initiations at the same sites. We found that branches extended (and retracted) at least once within 1 or 2 pixels of the location of 21 of the selected initiation events (not shown), supporting our conjec- ture that branches tend to recur with a high probability at or near certain positions along processes of $\mathrm{P}_{\mathrm{D}}$ neurons.

\section{Specific changes reflect process stabilization}

It would appear from the example discussed in the previous section that newly formed processes are quite unstable, undergoing several rounds of extension and full retraction, and have relatively short lifetimes $(\sim 8 \mathrm{~min})$. In this highly dynamic state, how does the arbor enlarge? Several measurable phenomena may indicate increasing process stability: longer lifetime (i.e., time for a complete extension-retraction cycle), incomplete retraction, and greater complexity.

To obtain a measure of cycle time $(\Delta t)$ and length changes $(\Delta l)$ in a population of processes, we measured the lengths of 50 randomly selected second- and third-order processes from two separate recording series, through one cycle of extension and retraction. Four images from one of these series are shown in Figure 8 . The average cycle time $\Delta t$ was approximately $14.3 \pm 6.8$ min (see Fig. $7 B$ ). This was a significantly longer period than the $8.1 \mathrm{~min}$ that we measured for only newly formed processes (see above). With respect to length change, there also was a slightly greater average extension than retraction $\left(\Delta l_{\text {ext }}>\Delta l_{\text {ret }}\right)$ in this sample (Fig. $9 A$; the linear regression lies above the extension equals retraction line). Over many cycles, this slight difference would presumably yield net process growth.

Extension rates for these 50 processes ranged from 0.25 to approximately $7.0 \mu \mathrm{m} / \mathrm{min}$, with average values of approximately 


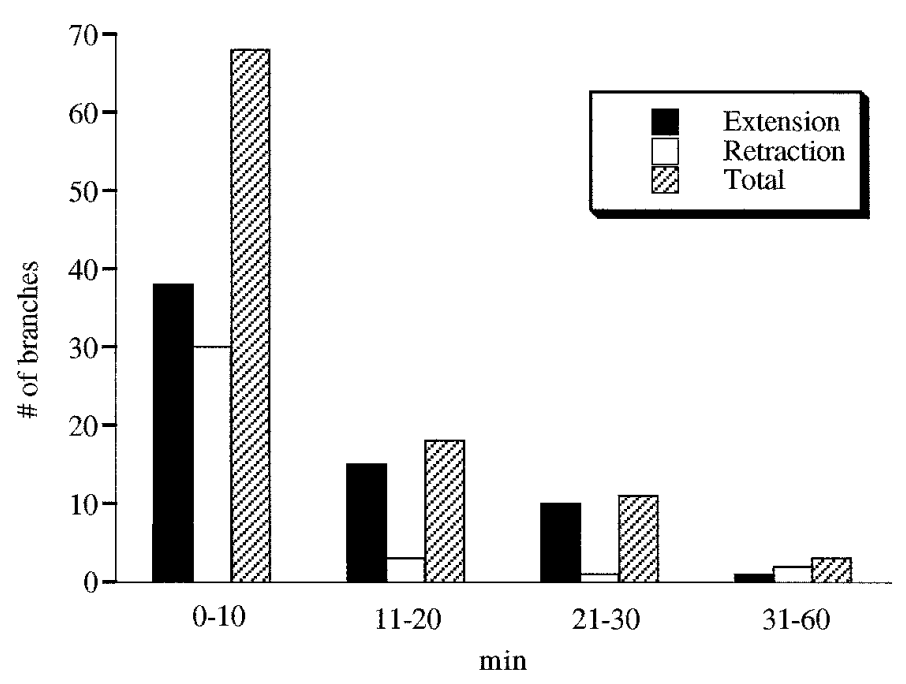

Figure 4. High-order branches are very dynamic. In a randomly selected sample of 100 branches, most extend or retract within $30 \mathrm{~min}$ of the beginning of the observation period. By $1 \mathrm{hr}$, all were observed to change their length. The histogram shows the number of branches that were found extending (black), retracting (white), or both (hatched) within the different time intervals indicated on the abscissa. For each of the 100 selected branches ( 25 from each of four different neurons), only the first time they either extended or retracted was recorded and counted in the corresponding time interval. Many of the branches changed their lengths multiple times during the $1 \mathrm{hr}$ observation period.

$2.3 \pm 1.2$ and $2.1 \pm 1.3 \mu \mathrm{m} / \mathrm{min}$ for extension and retraction, respectively. Thus, on average these processes extended at approximately the same velocity as they retracted. This is true for individual processes as well, as can be seen in the examples shown in Figure $9 B$, in which process length changes are plotted versus time. Interestingly, in many cases there is a pause of several minutes after maximum extension is attained and before retraction ensues (Fig. 9B, processes 3, 5, and 6). The nearly constant slopes seen in these plots also demonstrate that, for each branch, extension and retraction rates are nearly constant within each cycle.

In examining the time-lapse series, we also noted that some processes with branches appeared to be stable, not retracting completely over several hours, whereas others were not. Figure 10 gives an example of the retraction of a process with higher-order branches. To check whether having higher-order branches affected process stability, we randomly selected 100 processes, 25 in each of four separate recordings, and followed their development in the next $5 \mathrm{hr}$. Among these 100 processes, 14 that formed no higher-order branches during the $5 \mathrm{hr}$ period of observation withdrew completely. Of the other 86 , which all formed higherorder branches at least once during the observation, only 9 withdrew completely. Furthermore, among all processes that withdrew, those that formed higher-order branches lasted considerably longer on average than those that did not (Fig. 7C). In this sample, processes that had formed higher-order branches had a probability of retracting of only slightly $>10 \%$, whereas those that had no higher-order branches had a $100 \%$ probability of withdrawing. There is a positive correlation, therefore, between having higher-order branches and process stability, although processes with branches can and do sometimes retract completely.

\section{DISCUSSION}

How the terminal field of a $P_{D}$ neuron achieves its final complexity is an intriguing developmental problem. The approach we have taken, time-lapse imaging in the living, unanesthetized animal, reveals that this complexity is achieved, in part, through a very dynamic series of steps that includes repeated cycles of extension and retraction by most high-order branches. Previous studies of terminal arbor formation by the $\mathrm{P}_{\mathrm{D}}$ neuron in H. medicinalis (Gan and Macagno, 1995a) and by a similar sensory neuron $\left(\mathrm{P}_{\mathrm{V}}\right)$ in another species of leech (Haementeria ghilianii; Kramer and $\mathrm{Ku}$ wada, 1983; Kuwada and Kramer, 1983; Kramer et al., 1985), which were carried out using fixed preparations, lacked the temporal resolution necessary to detect this highly dynamic process.

The elaboration of the peripheral arbor of the $\mathrm{P}_{\mathrm{D}}$ neuron proceeds through several stages characterized by different morphologies and behaviors. First, after exiting the embryonic CNS, the efferent projection travels laterally along the inner surface of the forming body wall, along a pathway that eventually will include the medial dorsoventral muscle and the dorsal branch of the posterior nerve root. Whether the forming dorsoventral muscle (or any other element) plays a role in this directed outgrowth is not known. The projection has a large, elaborate growth cone with numerous filopodia, grows quickly to the dorsal target region, and does not branch noticeably in the underlying ventral territory.

Second, after reaching the target region in dorsal territory, the growth cone, still with many filopodia, continues to travel laterally to the edge of the dorsal body wall, where it pauses. In dorsal territory, however, in contrast to the behavior observed in ventral territory, major branches emanate from the axon shaft. These appear at specific positions behind the growth cone that, as reported in previous work, appear to correlate with the locations of particular longitudinal muscles (Gan, 1995; Gan and Macagno, 1995a). When examined in optical sections obtained with the confocal light microscope, these first-order branches can be observed to grow along and in close apposition to longitudinal muscle fibers (Fig. 2). First-order branches extend more slowly than the main projection, and lack elaborate growth cones.

Third, second-order branches emanate at various locations along first-order branches, from the growing tip to the point of origin. These branches undergo several cycles of extension and
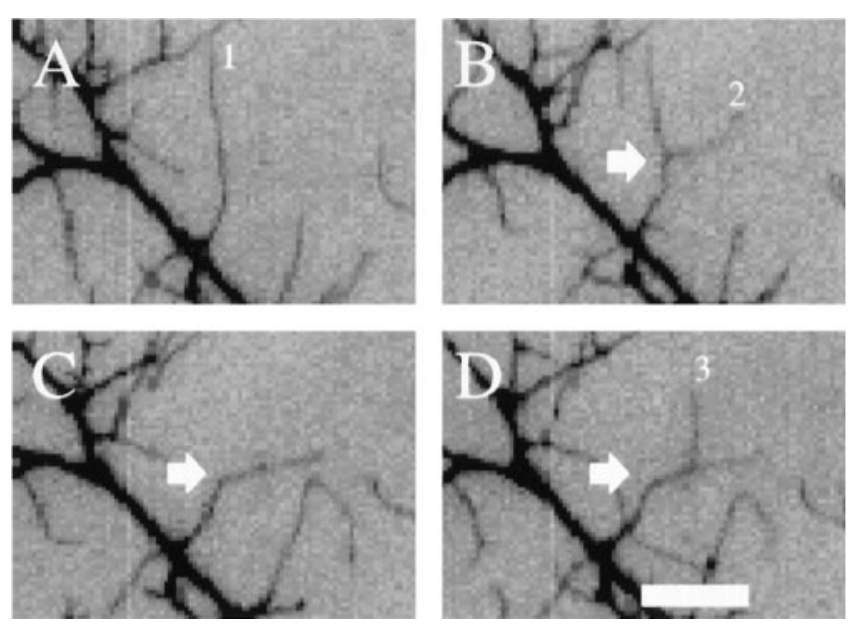

Figure 5. Branches can change their topological order. The figure shows four images from a time-lapse series at $0(A), 60(B), 145(C)$, and $222(D)$ min. The second-order process labeled 1 in $A$ has generated a third-order branch labeled 2 in $B$. The arrows in $B-D$ point to the bifurcation point. In $C$, process 1 has retracted to the bifurcation point, so that it now appears that processes 1 and 2 are part of a single, bent process. In $D$, a new, higher-order branch (3) has formed on the original third-order branch. Anterior is to the left. Scale bar for $A-D, 25 \mu \mathrm{m}$. 
A

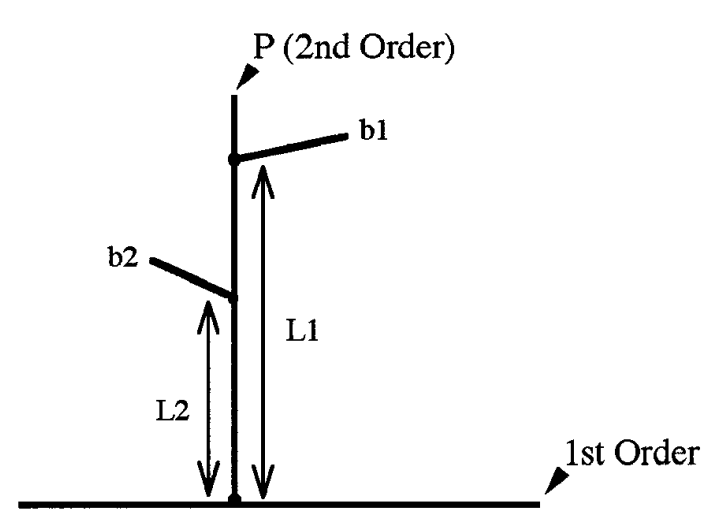

B

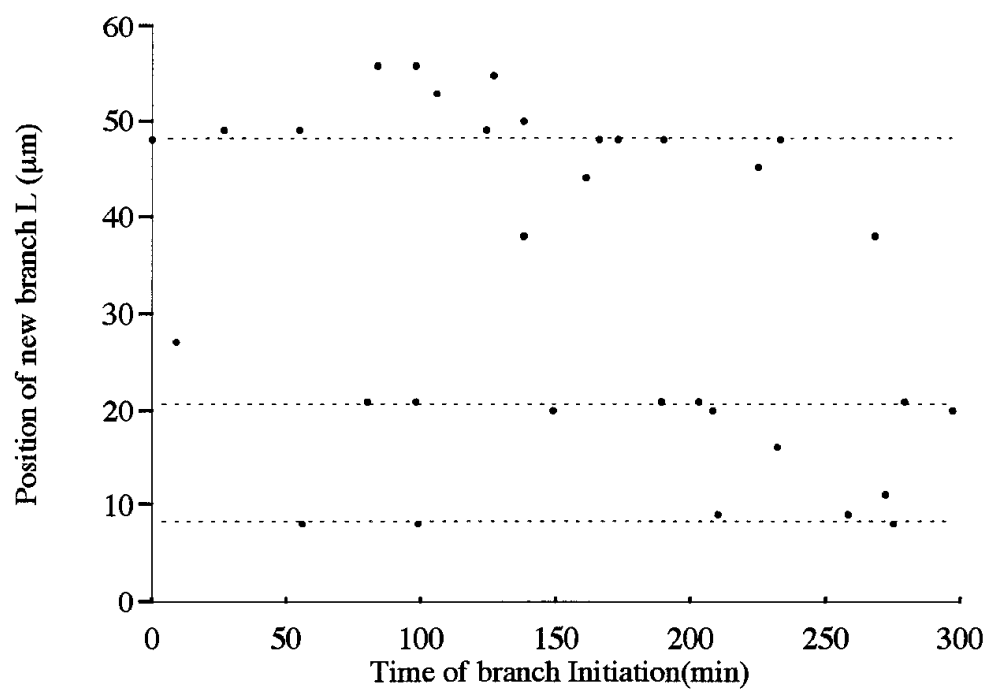

Figure 6. Branches tend to extend from hot spots along a parent process. $A$, Schematic drawing illustrating how positions of new branches were measured. Third-order branches $(b 1, b 2)$ are shown emanating from a second-order process that was considered as the parent process $(P)$ in these measurements. To position third-order branch initiation sites in the plot shown in $B$, the distances $(L 1, L 2)$ were measured from the point of origin of $\mathrm{P}$ (defined as zero) to the points of origin of these branches $(b 1, b 2)$ along P. $B$, Positions of the 33 new third-order branches generated by a selected parent process in a $300 \mathrm{~min}$ interval recorded in a time-lapse series, plotted against the time of their initiation. Horizontal dashed lines are drawn at the three positions along the parent process where there were multiple branch initiations.

retraction as they extend within a layer of unidentified cells toward the outer surface of the body wall. We found that to a large extent branches originate from particular locations on the parent process, which we termed "hot spots." However, unlike the first-order branches, the locations of second-order branches vary from neuron to neuron, indicating that they might not be associated with specific substrates that have consistent positions in the body wall.

Finally, in the last stage examined in these studies, the higherorder branches reach the circular muscle layer and appear to grow along and beyond these muscle fibers as they elaborate the sensory endings of the cell.

Many of the characteristics of axonal growth revealed by timelapse studies of very different types of neurons in other species are similar to those reported here for the growth of the $P_{D}$ neuron. Several recent time-lapse studies have examined the formation of retinotectal projections in semi-intact lower vertebrates. In Xenopus embryos, for example, retinal axons grow along the optic tract steadily and without branching, grow more slowly when their growth cones reach the tectum, and form their terminal arbors by back-branching behind the growth cone, these branches displaying small, simple growing tips (Harris et al., 1987). Interestingly, these axons seem to grow essentially normally for a few hours after they are severed from their cell bodies, suggesting that all of the machinery for responding to substrate cues resides in the growing region.

Terminal arbors of retinal axons, although much simpler than the arbor of the $\mathrm{P}_{\mathrm{D}}$ neuron, are also highly dynamic during early stages of their formation (Kaethner and Stuermer, 1992; O'Rourke et al., 1994). In Xenopus, a recent time-lapse study using hourly intervals showed that many branches had lifetimes shorter than $2 \mathrm{hr}$, with the rates of extension and retraction somewhat higher for the shorter branches (O'Rourke et al., 1994). Many other branches, however, were stable during the period of observation (up to $6 \mathrm{hr}$ ). A study in zebrafish at better time resolution (30 or $60 \mathrm{sec}$ intervals), however, suggests that forming retinal arbors are dynamic in the time scale of minutes (Kaethner and Stuermer, 1992), as is the arbor of the growing $P_{D}$ neuron.

Compared with the $\mathrm{P}$ cell terminal arbor, retinal arbors in the tecta of lower vertebrates are relatively small. In zebrafish, for example, retinal arbors are $\sim 20 \mu \mathrm{m}$ wide at early stages, which is less than the length of many individual high-order branches in the $\mathrm{P}$ cell terminal field. Another interesting difference is that the whole retinal arbor appears to shift as a result of remodeling; the area covered by an arbor at any one time was measured to be approximately one-sixth of the area touched by arbor processes, transient or stable, over the period of observation (Kaethner and Stuermer, 1992). By comparison, the $\mathrm{P}$ cell arbor expands without normally shifting its center of mass, although this can be induced experimentally (Gan and Macagno, 1995a).

Similar changes in the behavior of growing axons as they enter different regions along their path to and into the target region are also observed in developing callosal projections in hamster brain slices (Halloran and Kalil, 1994). In mouse CNS slice cultures, corticospinal axons have been observed to innervate their midbrain, hindbrain, and spinal targets by elaborating filopodia-like extensions (interstitial branches), some of them transient, well behind the growth cone (Bastmeyer and O'Leary, 1996). This behavior is quite reminiscent of the formation of branches by the $P_{D}$ neuron in its target region, and suggests that similar mechanisms may be present in these quite different systems.

Blocking synaptic transmission in the tectum with NMDA seems to decrease the stable population of branches in the retinal arbor (O'Rourke et al., 1994), suggesting that synaptic connection may be a factor in stabilizing the processes. In the arbor of the $\mathrm{P}$ cell, we have found that processes that generated higher-order processes were more stable, suggesting that the capacity for further branching is related to process stability. 

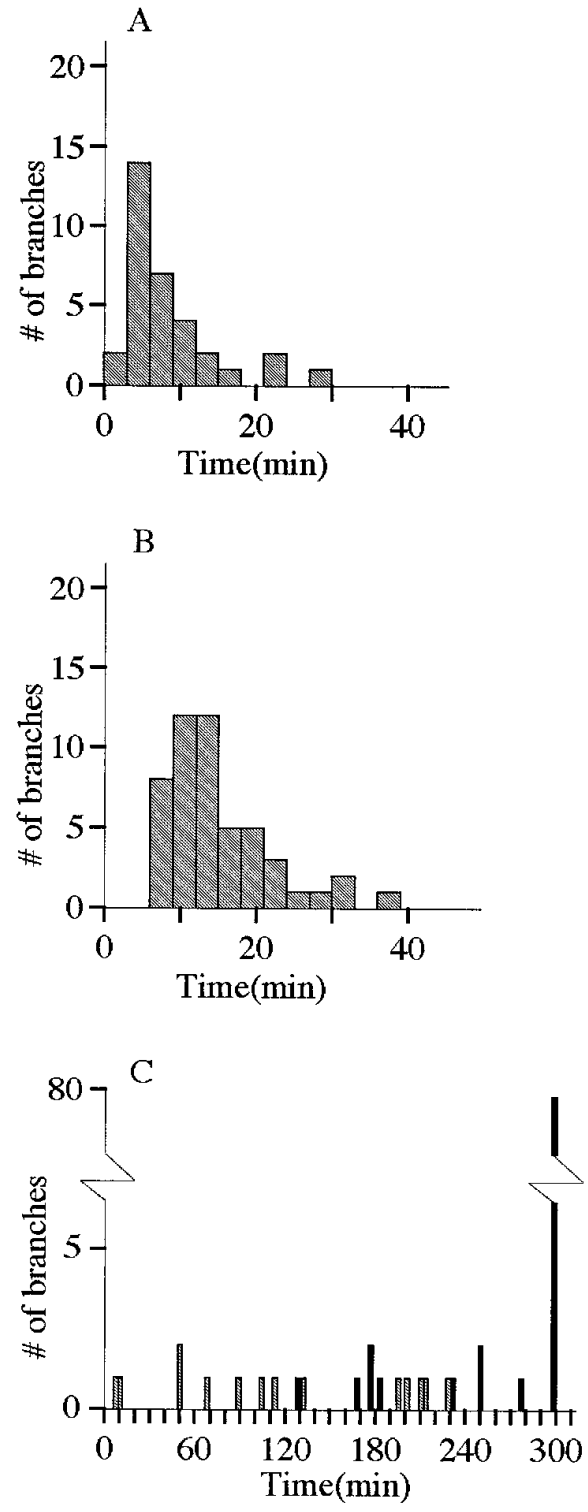

Figure 7. Data illustrating several dynamic variables of the terminal field. $A$, Lifetimes of a sample of 33 newly formed branches. The average lifetime is $8.1 \pm 6.2 \mathrm{~min}$ (mean $\pm \mathrm{SD}$ ). $B$, Full extension and retraction cycle time of a random sample of 50 branches. The average cycle time for this sample was $14.3 \pm 6.8 \mathrm{~min}$. $C$, The survival times, measured from the start of observation until full disappearance, of 100 randomly selected branches in a 300 min observation period. The survival times of those that generated higher-order branches during the observation period are shown in black, whereas those that did not generate higher-order branches are hatched. Most branches that generated higher-order branches (77 of 86) survived to the end of the $300 \mathrm{~min}$ observation period, whereas none survived among those that failed to generate them.

In our images, second- and higher-order branches initially look very much like the filopodia observed on the growth cone of the main projection of the $\mathrm{P}_{\mathrm{D}}$ neuron, although we would not be able to say whether they are the same diameter because this is below the resolution of the light microscope. Like filopodia in other systems, they have rapid extension and retraction rates of $\sim 100$ $\mu \mathrm{m} / \mathrm{hr}$ (Myers and Bastiani, 1993). Unlike a typical filopodium in a growth cone, however, which generally disappears as the growth cone passes the region, these processes of $\mathrm{P}$ cells can be much longer-lived. Some second-order processes have been observed to last for up to $10 \mathrm{hr}$ of recording, and are very likely to become permanent. In comparison, the long-lived filopodia on the growth cone of the $\mathrm{Q}_{1}$ neuron in the grasshopper only last approximately $1 \mathrm{hr}$ (Myers and Bastiani, 1993). Also, compared with a maximum length of $\sim 26 \mu \mathrm{m}$ for $\mathrm{Q}_{1}$ filopodia, the processes we studied could well exceed $60 \mu \mathrm{m}$.

Perhaps the most telling difference between filopodia and highorder processes of $\mathrm{P}_{\mathrm{D}}$ neurons is that the latter readily elaborate third- and higher-order branches, which is not characteristic of filopodia. Experiments currently under way to determine the cytoskeletal components of these very thin branches may help to resolve this question.

A consequence of the extensive cycling of processes, including the retraction of some that already have elaborated branches, is that the order of a process could change during development. A branch could retract to a bifurcation point and then leave a former higher-order branch, seemingly a part of a now longer process, as shown in Figure 5. This observation suggests that the topological order of individual processes in a branched arbor (Verwer and Pelt, 1986) may be surmised differently when looking at a static structure than it would be when the structure is examined dynamically.

The external and internal factors that determine the initiation position of higher-order branches along their parent process are unknown. It has been shown that diffusible factors might be involved in induction of new filopodia-like processes in other systems (Smith and Jahr, 1991; Sato et al., 1994). However, the observation that isolated mammalian retinal ganglion cells in vitro can generate an arbor pattern reminiscent of that in situ (Montague and Friedlander, 1991) suggests that there might be intrinsic mechanisms that specify dendritic branching patterns. Such mechanisms might be different, or nonexistent, for axon terminals. In the case of the $\mathrm{P}$ cells, because there appear to be hot spots along processes that are more likely to generate branches, it is possible that there are short-range external factors that influence the process initiation sites. The dual-channel imaging (Fig. 2) shows that there are undefined cells close to $\mathrm{P}$ cell processes that could make some locations along the parent process favorable or unfavorable for branch initiation. However, the existence of hot spots could also signify that the cytoskeletal change that occurs where a branch is first initiated is relatively permanent, and, hence, branches have a higher probability of recurring there.

Although our discussion has focused thus far on interactions of the $\mathrm{P}_{\mathrm{D}}$ cell processes with their environment, it is worth noting that there might be interactions between the hundreds of processes themselves. For example, there are sharp boundaries between the main field and the minor fields of an individual P cell, although $\mathrm{P}$ cell homologs residing in adjacent segments overlap extensively with one another (Kuffler and Muller, 1974; Gan and Macagno, 1995a). Previous developmental studies indicated that inhibition between sibling processes might be the cause of the sharp boundaries observed between main field and minor fields of a single cell (Kramer and Stent, 1985). Figure 8 shows a case in which two sibling processes overlap transiently and then retract. It is interesting to note that the processes do not stop or even slow down as they reach (and move transiently beyond) the boundary. To examine such possible interactions in detail requires a combination of time-lapse microscopy and three-dimensional imaging, which is currently in progress.

The results presented here demonstrate that the establishment 

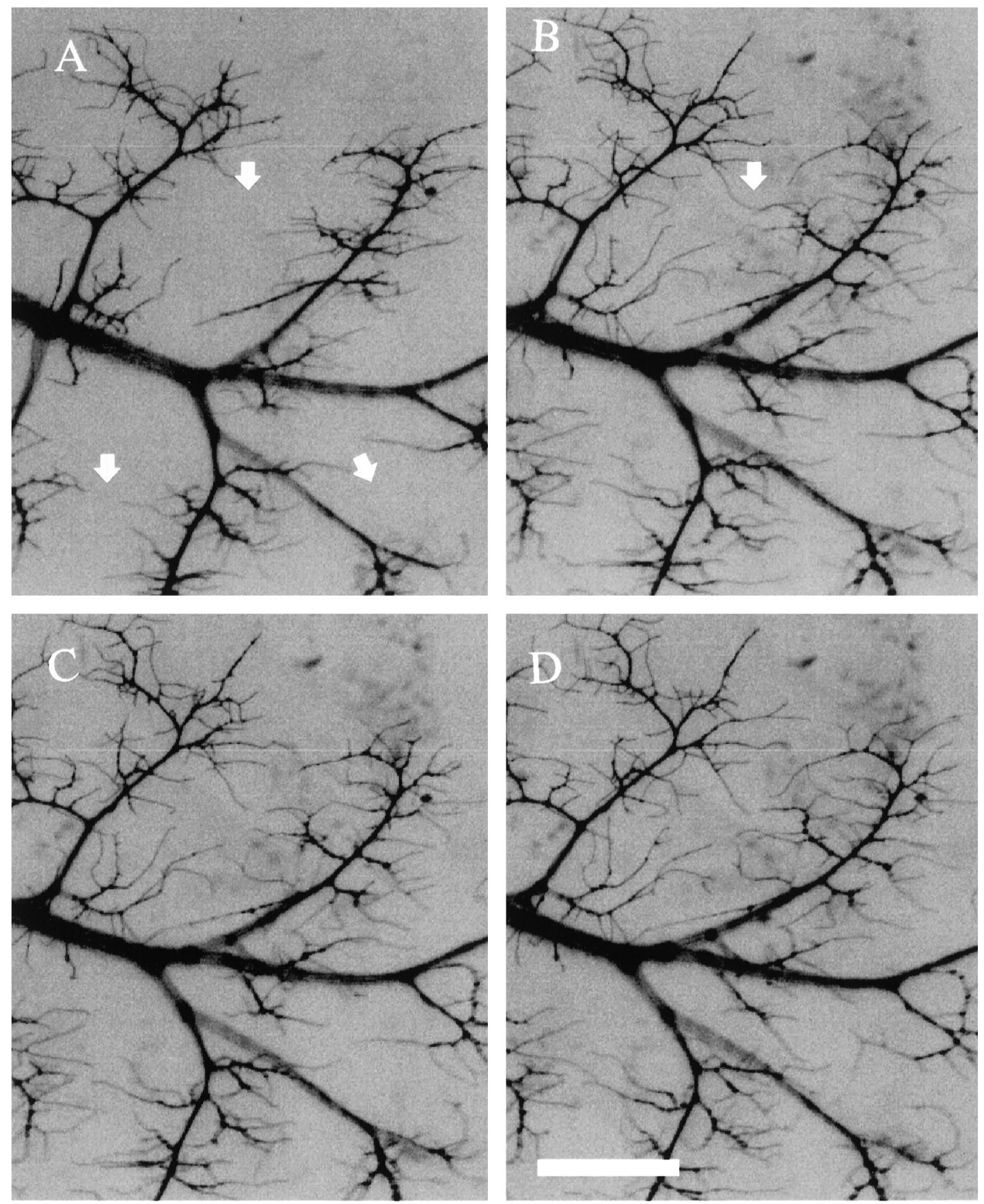

Figure 8. Subfields of primary branches fill the space between them, but without producing stable process overlaps. In this figure, anterior is $u p$. Four images from the 600 min time-lapse recording of a P cell arbor in a live E10 embryo are shown at $0(A), 452(B), 464(C)$, and $600(D)$ min. Arrows in $A$ point to unoccupied territories that are later filled by nearby second- and third-order processes. Arrow in $B$ indicates a point of overlap between two processes. This overlap disappeared a few minutes later, as shown in $C$, because of the retraction of one of the processes. Scale bar for $A-D$, $100 \mu \mathrm{m}$.

of a terminal field of a $\mathrm{P}_{\mathrm{D}}$ neuron is very dynamic. Our major findings include the following. (1) Most of the higher-order processes in the terminal fields are continually extending and retracting, even after reaching the target area. (2) The initiation of high-order processes along a parent branch is nonrandom, as there appear to be "hot-spots" for branch reinitiation. Terminal arbor formation has been described as "rapid remodeling" (O'Rourke at al., 1994) or "exploratory growth" (Kaethner and Stuermer, 1992). These terms best describe the behavior of the arbor as a whole. We suggest borrowing a term from the field of cytoskeleton research (Cassimeris, 1993), that the high-order processes within the $\mathrm{P}_{\mathrm{D}}$ cell arbor are in a state of "dynamic instability." Dynamic instability of microtubules describes a state in which individual microtubules are either extending or retracting and, in general, in which there is no steady state. This fits well with the behavior of the processes of the terminal arbor of the $P_{D}$ cell that we observed. Whether the dynamic instability of processes is a direct result of the dynamic instability of the cytoskeleton within the processes is a hypothesis we will be testing in subsequent studies of the $\mathrm{P}_{\mathrm{D}}$ cell. 
A

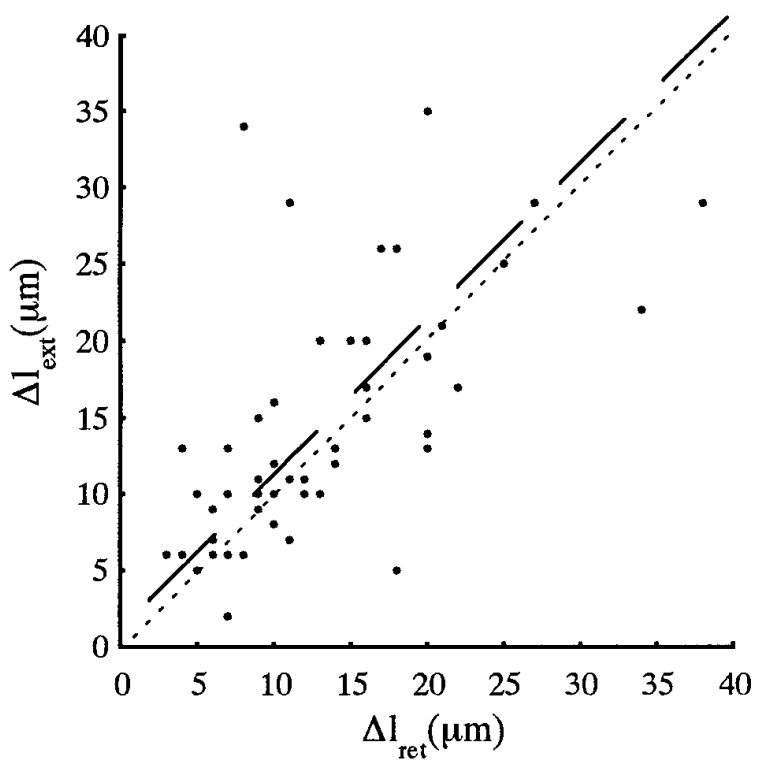

B

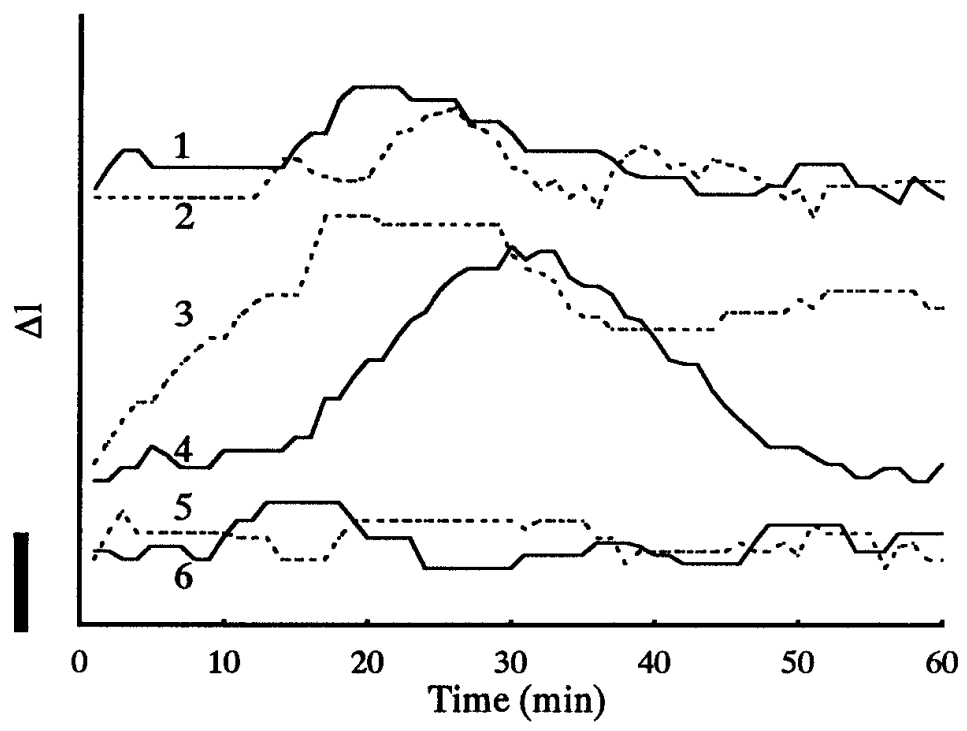

Figure 9. Individual processes extend and retract by similar amounts in each extension-retraction cycle. $A$, Extension lengths are plotted against the retraction lengths for 50 processes that underwent extension-retraction cycles. The data were taken from time-lapse recordings of two different preparations. The dotted line represents events in which the net length change is zero; events above this line represent net length gains, and events below represent net length losses. A best fit gives $\Delta l_{\text {ext }}=\Delta l_{\text {ret }}+1.3 \mu \mathrm{m}$, which is drawn as a dashed line. $B$, Changes in branch length versus time. The lengths of six branches were measured over a $60 \mathrm{~min}$ period, and changes were calculated and plotted against time. In general, during extension or retraction, changes in length appear to occur relatively smoothly and, for each process, at approximately the same absolute rate. Scale bar in $B, 20 \mu \mathrm{m}$.
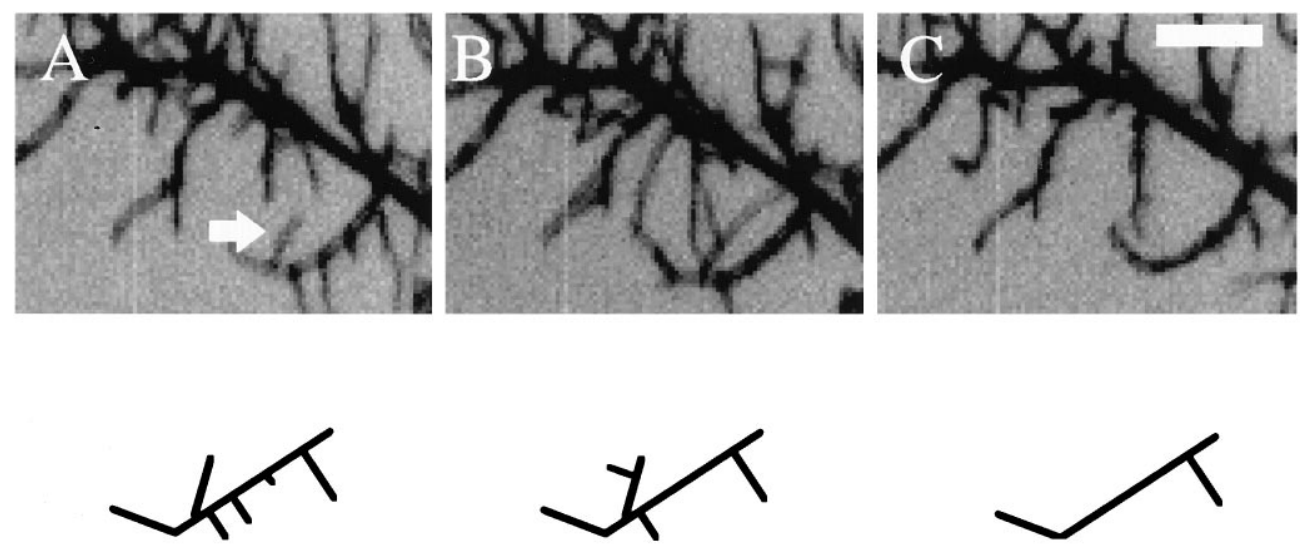

Figure 10. Processes with branches can be fully retracted. In this sequence of images from a time-lapse recording of an E10 preparation, the branch indicated by the white arrow in $A$ develops a higher-order branch $(B, 34$ min later) and is later fully retracted $(C, 134$ min later). The process of interest and its parent and higher-order branch are drawn schematically below the images. Note that other branches of the parent process are also retracted $(C)$. Scale bar for $A-C, 25 \mu \mathrm{m}$.

\section{REFERENCES}

Allen BA, Levinthal C (1990) CARTOS II semi-automated nerve tracing: three-dimensional reconstruction from serial section micrographs. Comput Med Imaging Graph 14:319-329.

Balice-Gordon RJ, Lichtman JW (1990) In vivo visualization of the growth of pre- and postsynaptic elements of neuromuscular junctions in the mouse. J Neurosci 10:894-908.

Bastmeyer M, O'Leary DD (1996) Dynamics of target recognition by interstitial axon branching along developing cortical axons. J Neurosci 16:1450-1459.

Cassimeris L (1993) Regulation of microtubule dynamic instability. Cell Motil Cytoskeleton 26:275-281.

Gan WB (1995) Cell-cell interactions regulate the formation of neuronal terminal fields. PhD dissertation, Columbia University, New York.

Gan WB, Macagno ER (1995a) Interactions between segmental ho- mologs and between isoneuronal branches guide the formation of sensory terminal fields. J Neurosci 15:3243-3253.

Gan WB, Macagno ER (1995b) Developing neurons use a putative pioneer's peripheral arbor to establish their terminal fields. J Neurosci 15:3254-3262.

Halloran MC, Kalil K (1994) Dynamic behaviors of growth cones extending in the corpus callosum of living cortical brain slices observed with video microscopy. J Neurosci 14:2161-2177.

Harris WA, Holt CE, Bonhoeffer F (1987) Retinal axons with and without their somata, growing to and arborizing in the tectum of Xenopus embryos: a time-lapse video study of single fibres in vivo. Development 101:123-133.

Jellies J, Kristan WB (1988) Embryonic assembly of a complex muscle is directed by a single identified cell in the medicinal leech. J Neurosci 8:3317-3326. 
Jellies J, Kristan WB (1991) The oblique muscle organizer in Hirudo medicinalis, an identified embryonic cell projecting multiple parallel growth cones in an orderly array. Dev Biol 148:334-354.

Kaethner RJ, Stuermer CA (1992) Dynamics of terminal arbor formation and target approach of retinotectal axons in living zebrafish embryos: a time-lapse study of single axons. J Neurosci 12:3257-3271.

Kramer AP, Kuwada JY (1983) Formation of the receptive fields of leech mechanosensory neurons during embryonic development. J Neurosci 3:2474-2486.

Kramer AP, Stent GS (1985) Developmental arborization of sensory neurons in the leech Haementeria ghilianii. II. Experimentally induced variations in the branching pattern. J Neurosci 5:768-775.

Kramer AP, Goldman JR, Stent GS (1985) Developmental arborization of sensory neurons in the leech Haementeria ghilianii. I. Origin of natural variations in the branching pattern. J Neurosci 5:759-767.

Kuffler DP, Muller KJ (1974) The properties and connections of supernumerary sensory and motor nerve cells in the central nervous system of an abnormal leech. J Neurobiol 5:331-348.

Kuwada JY, Kramer AP (1983) Embryonic development of the leech nervous system: primary axon outgrowth of identified neurons. J Neurosci 3:2098-2111.

Lichtman JW, Magrassi L, Purves D (1987) Visualization of neuromuscular junctions over periods of several months in living mice. J Neurosci 7:1215-1222.

Levinthal F, Macagno E, Levinthal C (1976) Anatomy and development of identified cells in isogenic organisms. Cold Spring Harb Symp Quant Biol 40:321-331.

Macagno ER, Lopresti V, Levinthal C (1973) Structure and development of neuronal connections in isogenic organisms: variations and similarities in the optic system of Daphnia magna. Proc Natl Acad Sci USA 70:57-61.

Miller M (1981) Maturation of rat visual cortex. I. A quantitative study of Golgi-impregnated pyramidal neurons. J Neurocytol 10:859-878.

Miller M (1986) Maturation of rat visual cortex. III. Postnatal morphogenesis and synaptogenesis of local circuit neurons. Dev Brain Res 25:271-285.

Montague PR, Friedlander MJ (1989) Expression of an intrinsic growth strategy by mammalian retinal neurons. Proc Natl Acad Sci USA 86:7223-7227.

Montague PR, Friedlander MJ (1991) Morphogenesis and territorial coverage by isolated mammalian retinal ganglion cells. J Neurosci 11:1440-1457.
Muller KJ, McMahan UJ (1976) The shapes of sensory and motor neurons and the distribution of their synapses in the ganglia of the leech: a study using intracellular injection of horseradish peroxidase. Proc R Soc Lond [Biol] Sci 194:481-499.

Myers PZ, Bastiani MJ (1993) Growth cone dynamics during the migration of an identified commissural growth cone. J Neurosci 13:127-143.

Nicholls JG, Baylor DA (1968) Specific modalities and receptive fields of sensory neurons in CNS of the leech. J Neurophysiol 31:740-756.

O'Connor TP, Duerr JS, Bentley D (1990) Pioneer growth cone steering decisions mediated by single filopodial contacts in situ. J Neurosci 10:3935-3946.

O'Rourke NA, Cline HT, Fraser SE (1994) Rapid remodeling of retinal arbors in the tectum with and without blockade of synaptic transmission. Neuron 12:921-934.

Peters A, Jones EG (1984) Cerebral cortex. In: Cellular components of the cerebral cortex, Vol 1. New York: Plenum.

Regehr WG, Tank DW (1992) Calcium concentration dynamics produced by synaptic activation of CA1 hippocampal pyramidal cells. J Neurosci 12:4202-4223.

Sargent PB, Yau KW, Nicholls JG (1977) Extrasynaptic receptors on cell bodies of neurons in central nervous system of the leech. J Neurophysiol 40:446-452.

Sato M, Lopez-Mascaraque L, Heffner CD, O'Leary DD (1994) Action of a diffusible target-derived chemoattractant on cortical axon branch induction and directed growth. Neuron 13:791-803.

Smith SJ, Jahr CE (1991) Rapid induction of filopodial sprouting by application of glutamate to hippocampal neurons. In: The nerve growth cone (Letourneau PC, Kater SB, Macagno ER, eds), pp 19-26. New York: Raven.

Solomon F (1979) Detailed neurite morphologies of sister neuroblastoma cells are related. Cell 16:165-169.

Verwer RWH, Pelt J (1986) Descriptive and comparative analysis of geometrical properties of neuronal tree structures. J Neurosci Methods 18:179-206.

Wenning A (1987) Salt and water regulation in Macrobdella decorata (Hirudinea: Gnathobdeliformes) under osmotic stress. J Exp Biol 131:337-349.

Yau KW (1976) Receptive fields, geometry and conduction block of sensory neurons in the central nervous system of the leech. J Physiol (Lond) 263:513-538. 